\title{
Revising ventricular septal defect residual shunts without aortic re-cross-clamping: a safe and effective surgical procedure
}

\author{
Zhongming Cao ${ }^{1 \#}$, Yunfei Chai ${ }^{1 \#}$, Jian Liu ${ }^{2 \#}$, Shiguo Liu ${ }^{1}$, Jinfeng Wei ${ }^{1}$, Jiexian Liang ${ }^{1}$, Jian Zhuang ${ }^{2}$, \\ Sheng Wang ${ }^{1}$, Gang $\mathrm{Xu}^{2} \wedge$ \\ ${ }^{1}$ Department of Anesthesiology, Guangdong Provincial Cardiovascular Institute, Guangdong Provincial People's Hospital/Guangdong Academy \\ of Medical Sciences, Guangzhou, China; ${ }^{2}$ Department of Cardiac Surgery, Guangdong Provincial Cardiovascular Institute, Guangdong Provincial \\ People's Hospital/Guangdong Academy of Medical Sciences, Guangzhou, China \\ Contributions: (I) Conception and design: Z Cao, Y Chai, J Liu; (II) Administrative support: S Wang, G Xu; (III) Provision of study materials or \\ patients: J Liang, S Wang, G Xu; (IV) Collection and assembly of data: Z Cao, S Liu; (V) Data analysis and interpretation: Z Cao, Y Chai, J Wei; (VI) \\ Manuscript writing: All authors; (VII) Final approval of manuscript: All authors. \\ \#These authors contributed equally to this work. \\ Correspondence to: Gang Xu, MD, PhD. Department of Cardiac Surgery, Guangdong Provincial Cardiovascular Institute, Guangdong Provincial \\ People's Hospital/Guangdong Academy of Medical Sciences, Guangzhou, China. Email: xuganghjj@163.com; Sheng Wang, MD, PhD. Department \\ of Anesthesiology, Guangdong Provincial Cardiovascular Institute, Guangdong Provincial People's Hospital/Guangdong Academy of Medical \\ Sciences, Guangzhou, China. Email: shengwang_gz@163.com.
}

Background: The conventional approach to revising a residual shunt following ventricular septal defect (VSD) closure is to re-occlude the aorta and repair the residual shunt under cardioplegic arrest. The present study evaluated the safety and effectiveness of a new approach for revising residual shunts following VSD repair without re-occluding the aorta. This approach is known as on beating heart surgery.

Methods: This retrospective study included 80 pediatric patients who underwent surgical closure of a simple VSD. Residual shunts larger than $2 \mathrm{~mm}$ were intraoperatively detected by transesophageal echocardiography (TEE) and these patients received immediate reintervention. Of the patients, 37 received on beating heart surgery without aortic cross-clamping (Group A) and 43 patients were operated on with aortic cross-clamping and cardioplegia (Group B).

Results: Residual VSD closures were successfully performed in all patients. Group A had significantly shorter aortic cross-clamp times $(\mathrm{P}<0.0001)$, significantly shorter $\mathrm{CPB}$ times $(\mathrm{P}<0.01)$, a lower incidence of prolonged ventilation ( $>6$ hours) $(\mathrm{P}=0.04)$, a lower incidence of prolonged intensive care unit (ICU) stay (ICU stay $>1$ day) $(\mathrm{P}=0.02)$, and reduced in-hospital expenses $(\mathrm{P}<0.0001)$ compared with Group B. There was no significant difference in the incidence of recurrent residual shunts $(\mathrm{P}=0.96)$, prolonged postoperative hospital stay ( $>5$ days) $(\mathrm{P}=0.24)$, or the incidence of perioperative complications $(\mathrm{P}=0.81)$ between the groups.

Conclusions: On beating heart surgery is a safe and effective approach for the closure of residual VSDs and is significantly associated with a lower incidence of prolonged ventilation, a lower incidence of prolonged ICU stay, and reduced in-hospital expenses.

Keywords: Residual ventricular septal defect (residual VSD); beating heart; cardioplegic arrest; aortic cross-clamp

Submitted Jun 08, 2020. Accepted for publication Sep 01, 2020.

doi: 10.21037/atm-20-5041

View this article at: http://dx.doi.org/10.21037/atm-20-5041

^ Zhongming Cao, ORCID: 0000-0001-6423-8083; Gang Xu, ORCID: 0000-0003-0753-4032. 


\section{Introduction}

Ventricular septal defect (VSD) is the most common congenital cardiac anomaly during fetal life and at birth, accounting for approximately $20-30 \%$ of congenital heart malformations (1,2). In the 1950s, Gibbon et al. successfully repaired ASDs using cardiopulmonary bypass techniques (3), followed by the rapid development of surgical techniques for other congenital heart diseases, including VSDs. Although percutaneous transcatheter approaches have been used extensively in the treatment of VSDs, surgical closure by standard median sternotomy with direct visualization of the defect is the most common pediatric cardiac operation $(4,5)$. However, residual shunts are detected by intraoperative transesophageal echocardiography (TEE) and postoperative transthoracic echocardiography (TTE) in 9-36\% of patients following surgical closure of a VSD (6,7). Residual VSDs smaller than $2 \mathrm{~mm}$ are mild and tend to close spontaneously in most patients within a year. Residual defects larger than $2 \mathrm{~mm}$, however, require continuous surveillance and may require reintervention (8). Many studies have also indicated that the risk of developing infective endocarditis is significantly higher in patients with untreated VSDs. Intraoperative assessment of residual shunts larger than $2 \mathrm{~mm}$ detected during cardiopulmonary bypass (CPB) and initial VSD repair may therefore prevent the need for reintervention and lifelong antibiotic prophylaxis (9).

If a severe residual shunt is detected by TEE intraoperatively after removal of the aortic cross-clamp, the conventional approach involves re-occlusion of the aorta under cardioplegic arrest. Although this approach provides an adequate surgical field of view that allows for unobstructed suturing, cross-clamping the aorta again increases the risk of adverse events, including myocardial ischemia-reperfusion injury (10). Aortic cross-clamp time is a determinant of late events, particularly in children (11). Surgeons at our cardiovascular center have conceived a new approach for revising residual shunts in certain pediatric patients. This involves closing the residual shunt directly on the beating heart and does not involve aortic re-crossclamping. However, research comparing this new approach with conventional residual shunt treatment in children is limited (12). This retrospective study compares the shortterm and medium-term outcomes of these two methods to evaluate the relative significance of this novel on beating heart approach for revising residual shunts.

We present the following article in accordance with the
STROBE reporting checklist (available at http://dx.doi. org/10.21037/atm-20-5041).

\section{Methods}

\section{Patient selection}

The study was conducted in accordance with the Declaration of Helsinki (as revised in 2013), and reviewed and approved by the Institutional Review Board of Guangdong Province People's Hospital (Guangzhou, Guangdong, China) (No. GDREC2019768H) on 31 December, 2019. The requirement for informed consent was waived because of the retrospective nature of the research. This study analyzed the medical records of 5,631 pediatric surgical patients ( $<14$ years old) who underwent surgical closure of a simple VSD and presented to the Guangdong Province People's Hospital between March, 2011, and August, 2019. A simple VSD was clinically defined as an isolated VSD or a VSD associated with other congenital heart defects (CHD), such as atrial septal defects, patent foramen ovale, patent ductus arteriosus, and mildly stenotic and regurgitant semilunar valves (13). The primary indication for surgical treatment in all patients was VSD closure. Ninety-three (1.65\%) patients with a VSD residual shunt of $2-5 \mathrm{~mm}$ detected by intraoperative TEE required their CPB to be resumed and the shunt repaired. To keep the preoperative clinical characteristics and patient demographics of the two groups as similar as possible, patients meeting any of the following criteria were excluded: (I) a preoperative weight of $<4 \mathrm{~kg}$; (II) aged $<1$ month at time of surgery; (III) cardiac failure; (IV) pulmonary infection; (V) down syndrome; or (VI) other co-morbidities $(13,14)$. Eighty patients met the inclusion criteria and were included in the study; of these patients, 37 had their residual shunts revised on beating hearts without aortic cross-clamping (Group A), and 43 patients were operated on with aortic cross-clamping under cardioplegic arrest (Group B).

\section{Surgical procedure}

All operations were performed by three experienced surgeons of similar seniority. Using standard surgical techniques, $\mathrm{CPB}$ was performed with moderate hypothermia $\left(30^{\circ} \mathrm{C}\right)$ following cross-clamping of the aorta and cold crystal cardioplegic arrest. Perimembranous VSDs were accessed through an incision to the right atrium, while subarterial VSDs were accessed through an incision 
to the pulmonary artery. VSDs were then repaired using autologous pericardial patches with continuous sutures. TEE was routinely performed on all patients after aortic unclamping; Philips iE33 and Philips iE Elite ultrasound systems (Philips Medical Systems, Netherlands) were used. However, when residual shunts between 2-5 $\mathrm{mm}$ were located by TEE, the surgeon immediately re-opened the heart through the original incision to achieve residual defect closure.

Patients treated between March, 2011 and May, 2015 had residual shunts repaired with aortic cross-clamping and cardioplegic arrest (Group B). Patients treated between June, 2015, and August, 2019, had residual shunts repaired on beating hearts without aortic cross-clamping (Group A). The position of the residual shunt in Group A patients was located via TEE; jet blood flow appeared at the orifice during cardiac contractions. Patches were then directly sutured over the shunt with $0 / 4$ prolene until no obvious jet blood flow remained at the orifice. Residual shunts in the Group B patients were also located by TEE. After the heart was opened, the suspected shunt position was detected with a nerve hook to determine the gap. This was then sutured. If a residual shunt could not be confirmed, the patch was removed and the shunt sutured again. To further reduce the risk of air embolism in the Group A patients, the operating field was continuously flushed with carbon dioxide. Patients were also placed in the Trendelenburg position to prevent air from entering the brain, to maintain high aortic pressure $(60 \mathrm{mmHg}$ or above) and reduce heart ejection, and to exert appropriate negative pressure $(-10$ to $-30 \mathrm{mmHg})$ in the suction tube at the aortic root to suck out bubbles from the left ventricle. Intraoperative TEE was performed continually to monitor the left ventricle and the anesthesiologist watched for changes in the electrocardiograph (ECG) as the surgeon revised the residual shunt. If a patient's heart rate dropped below $60 \mathrm{bpm}$, accompanied by QRS broadening and a complete atrioventricular blockage, the surgeon was reminded to adjust the position of the sutures until the ECG recovered. After the aorta was opened for the second time, TEE was reexamined to rule out residual VSD. Residual shunts larger than $2 \mathrm{~mm}$ could not be tolerated and required repair again.

\section{Patient follow-up}

Patients were monitored postoperatively in the intensive care unit (ICU). TTE was performed routinely before patient discharge and during follow-up to assess heart function and the cardiac structures. Follow-up data were collected from hospital records and included survival after discharge, readmission, and TTE and ECG findings. All patients were followed up for 12 months after discharge and their status at the time of our study was confirmed by telephone.

\section{Statistical analysis}

Statistical analysis was performed using SPSS 21.0 software (IBM, Armonk, NY, USA). Continuous variables with a normal distribution were expressed as mean [standard deviation (SD)], and non-normal variables were presented as median [interquartile range (IQR)]. The differences between the two groups were assessed with the unpaired $t$-test. A Mann-Whitney $\mathrm{U}$ test was used to compare the means of variables not normally distributed between the two groups. The frequencies of categorical variables were compared using the chi-squared test. A value of $\mathrm{P}<0.05$ was considered statistically significant.

\section{Results}

\section{Patient characteristics}

The patients' preoperative clinical and demographic characteristics are summarized in Table 1. During the early phase of this study, this new method was performed on older children of greater body weight; the procedure was later applied to younger and lighter children. The mean age and weight of Group A were slightly lower than that of Group B; however, the difference between the two groups was not significant $(\mathrm{P}>0.05)$. Other baseline characteristics of the two groups were reasonably similar.

\section{Perioperative parameters}

Perioperative parameters are presented in Table 2. Compared with Group B, aortic cross-clamp time in Group A was shortened by $55 \%$ and CPB time was shortened by $38 \%$; the differences between the two groups were statistically significant $(\mathrm{P}<0.0001)$. The incidences of prolonged mechanical ventilation and extended duration of ICU stay were significantly lower in Group A compared with Group B $(\mathrm{P}<0.05)$. The median in-hospital expenses were significantly lower for Group A patients $(36,725$ RMB¥) than for Group B patients (43,112 RMB¥) $(\mathrm{P}<0.0001)$. However, there was no significant difference 
Table 1 Patient demographic characteristics

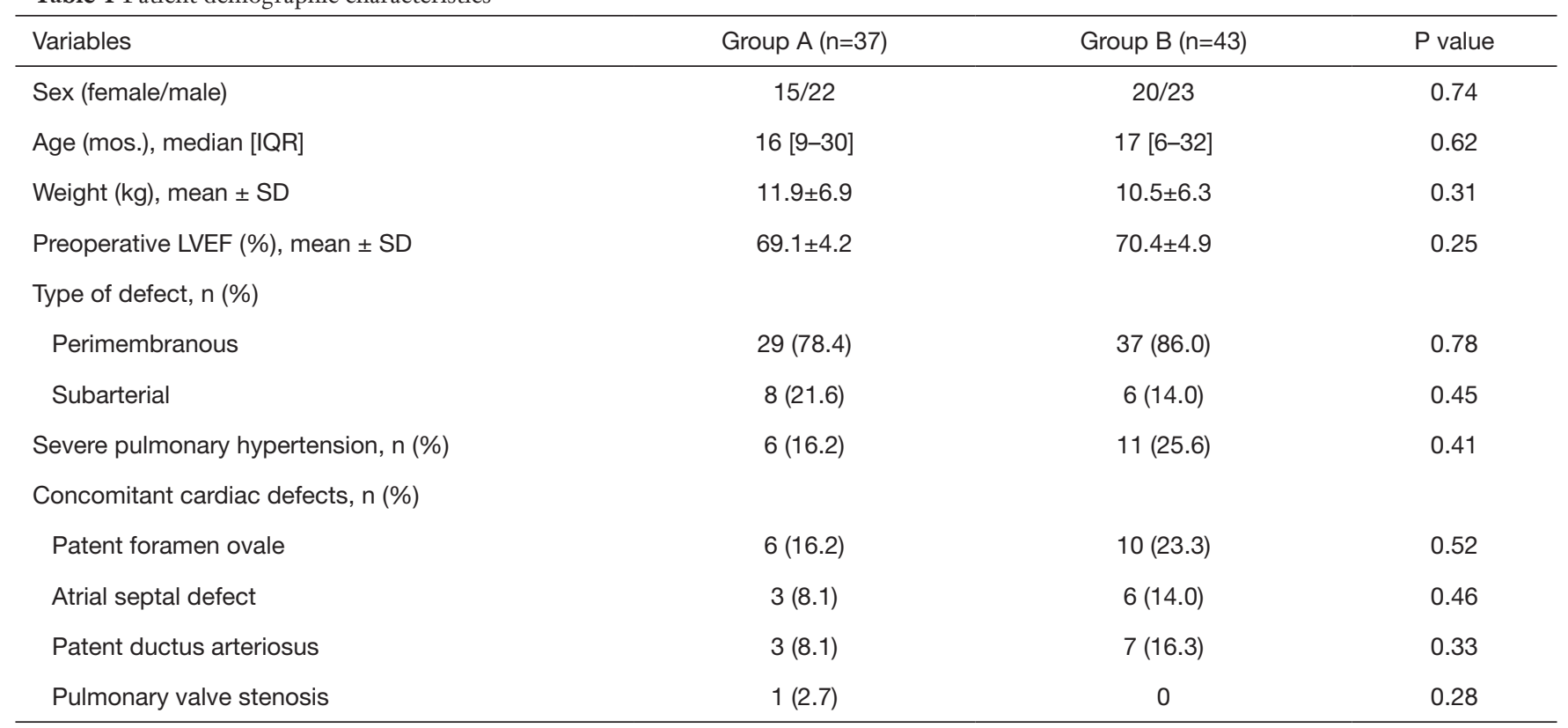

Group A patients received on beating heart surgery without aortic cross-clamping; Group B patients received surgery with aortic crossclamping and cardioplegia; LVEF left ventricular ejection fraction.

between the two groups in the proportion of patients who received higher doses of vasoactive drugs and isoproterenol, the incidence of prolonged postoperative hospital stay, and the development of complications. Perioperative complications experienced by the patients are presented in Table 3. During the perioperative period, no patients exhibited severe postoperative complications, such as the inability to withdraw extracorporeal circulation, excessive bleeding requiring reoperation, epilepsy, or death.

\section{The results of postoperative echocardiography}

The number and percentage of patients with different residual shunt sizes at different times, as detected by TEE, are shown in Table 4. The size of residual shunts following the first aortic unclamping was $2-5 \mathrm{~mm}$ in both groups. Residual VSDs were repaired successfully immediately in most patients; 1 patient in Group A and 2 patients in Group B exhibited mild residual shunts as shown by TTE at their last follow-ups. There was no significant difference in the proportion of patients remain residual shunt after reoperation between the two groups.

\section{Evaluation of new TR and abnormal ECG}

New cases of tricuspid regurgitation (TR) and abnormal
ECG results are shown in Table 5. Postoperative TEE monitoring revealed 2 patients in Group A and 3 patients in Group B with mild TR (regurgitation area $<20 \%$ of the right atrial area). In Group A, 1 patient presented with moderate TR (20-40\% regurgitation region in the right atrial area) on pre-discharge TTE that persisted until the next follow-up. One patient in Group B presented with moderate TR before discharge that reduced to mild TR during the follow-up period. Frequent ventricular premature beats (FVPB) were detected in 1 patient in Group A; 1 patient exhibited complete atrioventricular block (CAVB) in Group B; and 1 patient presented with paroxysmal supraventricular tachycardia (PST) in Group B. All of these patients were stabilized before discharge. Another significant ECG anomaly was complete right bundle branch blocks (CRBBB); however, there was no significant difference in the proportion of newly occurring TR and abnormal ECG between the two groups. At the time of final follow-up, all of the patients were alive and had not required readmission.

\section{Discussion}

The conventional approach to revising residual VSDs requires the aorta to be re-occluded under cardioplegic arrest. However, this re-occlusion and the resultant 
Table 2 Perioperative parameters

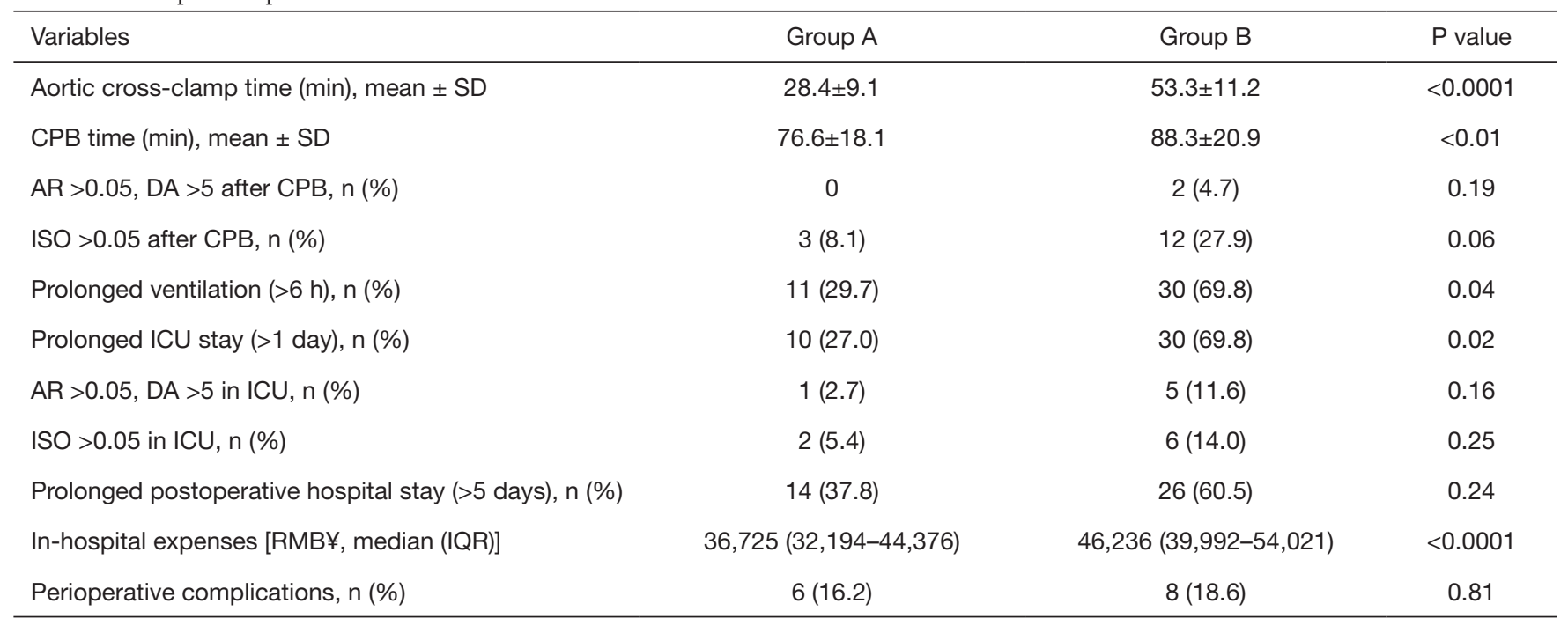

ICU, intensive care unit; CPB, cardiopulmonary bypass; AR, adrenaline; DA, dopamine; ISO, isoproterenol. The rate of administration is $\mu \mathrm{g} / \mathrm{kg} / \mathrm{min}$.

Table 3 Perioperative complications

\begin{tabular}{lcc}
\hline Complications & Group A (n) & Group B (n) \\
\hline Pulmonary infection & 2 & 3 \\
Poor wound healing & 2 & 2 \\
Massive pleural effusion & 1 & 0 \\
Pulmonary atelectasis & 1 & 2 \\
Diaphragmatic paralysis & 0 & 1 \\
\hline
\end{tabular}

prolonged occlusion of the aorta increases the risk of ischemia-reperfusion injury to the myocardium $(11,15)$. The risk of damage to the myocardial valve and bundle also increases with the removal of the pericardial patch and subsequent revision $(10,12,16)$. Additionally, if cardioplegia is induced again, taking into account the cooling and rewarming processes, CPB time would also be prolonged. Prolonged CPB time results in the destruction of blood elements, systemic inflammation, electrolyte imbalance, myocardial edema, and pulmonary complications, all of which affect patient prognosis following surgical intervention (17). Apparently, revising a VSD residual shunt on a beating heart avoids aortic re-occlusion and shortens CPB time. Additionally, the location of a residual shunt can be approached more intuitively on a beating heart, and damage to the conduction bundle can be avoided by observing the ECG. However, many surgeons are apprehensive of using this technique in routine practice due to concerns of air embolism and the difficulty involved with suturing a beating heart.

In a previous study, Ozal et al. compared the records of 17 adult patients who had the lower edge of their VSDs repaired on beating hearts after aortic unclamping and 158 patients who had their VSD repaired by aortic crossclamping and cardioplegic arrest. Notably, the incidence of residual shunt and CAVB in the former group was significantly reduced compared to the latter, without the presence of air embolism, neurological complications, or death (12). Yilmaz et al. examined 87 patients after opening the aorta for VSD repair, looking for any residual shunt on the beating heart; these were immediately repaired on the beating heart. They found it was advantageous for detecting and repairing any residual shunts in a timely fashion (18). Other studies that have investigated VSD repair on beating hearts have included patients that presented with VSD after acute myocardial infarction $(16,19,20)$. In these cases, repairing septal defects on beating hearts helped to prevent myocardial ischemia-reperfusion injury in patients with reduced cardiac function. However, these previous studies were on adult VSD patients, and they did not compare the effect of the two techniques on postoperative ICU stay, length of hospital stay, inpatient complications, and shortterm and medium-term outcomes. In this context, Tang et al. compared the on beating heart and cardioplegic techniques 
Table 4 The results of postoperative echocardiography

\begin{tabular}{|c|c|c|c|c|}
\hline Variables & Characteristics of residual VSD & Group A, n (\%) & Group B, n (\%) & $P$ value \\
\hline First postoperative TEE & $3-5 \mathrm{~mm}$ & $15(40.5)$ & $16(37.2)$ & 0.84 \\
\hline Second postoperative TEE & $<2 \mathrm{~mm}$ & $5(13.5)$ & $6(14.0)$ & 0.96 \\
\hline Pre-discharge TTE & $<2 \mathrm{~mm}$ & $6(16.2)$ & $6(14.0)$ & 0.81 \\
\hline Follow-up TTE & $<2 \mathrm{~mm}$ & $1(2.7)$ & $2(4.7)$ & 0.66 \\
\hline
\end{tabular}

TEE, transesophageal echocardiography; TTE, transthoracic echocardiography.

Table 5 Evaluation of new TR and abnormal ECG

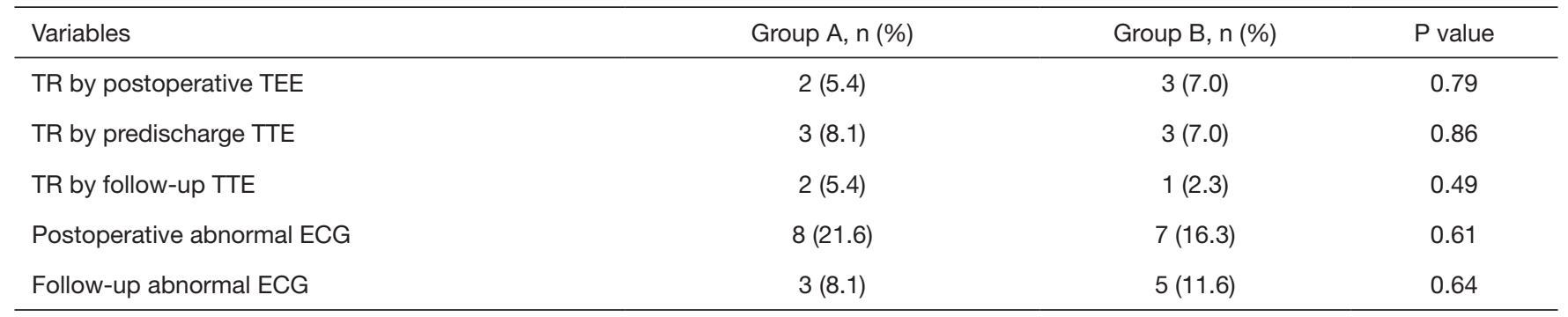

TR, tricuspid regurgitation; ECG, electrocardiograph.

in the repair of atrial septal defects (ASDs) and found that the durations of surgery, $\mathrm{CPB}$, aortic cross-clamp, mechanical ventilation, ICU stay, and postoperative hospital stay were all markedly shorter in the on beating heart group $(\mathrm{P}<0.05)$ and no severe complications occurred $(21)$. In the present study, residual VSD closures were successfully performed in all pediatric patients in both Groups A and B without associated in-hospital mortality or other severe complications. Notably, residual shunts were quickly found and repaired on the beating heart, which significantly reduced not only aortic cross-clamp time and $\mathrm{CPB}$ time, but also the incidence of prolonged postoperative ventilation and prolonged ICU stay. Previous studies have shown that this significant reduction in aortic crossclamping time and CPB time may significantly contribute to shortened ventilation time and ICU stays (13). When we reviewed patients' histories, we found that the prolonged ICU stays in Group B were primarily due to postoperative complications. Although there was no significant difference in the incidence of postoperative complications between the two groups, Group B patients with postoperative complications had longer ICU stays than Group A patients with postoperative complications. This directly accounted for the difference in hospitalization costs between the two groups.

Despite concerns that operating on a beating heart may result in accidental heart tissue damage, our study indicated that this approach did not increase the risk of associated complications. Heart surgery in pediatric patients involves elaborate procedures; however, we encountered no difficulty in performing these surgeries. With regard to the probability of secondary residual shunts, there were not significantly more TR and ECG abnormalities in Group A than Group B. CAVB has been found to occur in $0.9 \%$ to $4 \%$ of patients following VSD repair surgery; these patients require permanent pacemakers (22-24). CAVB is thought to occur following surgery as a result of the sutures in the atrioventricular bundle. To avoid this, as the surgeon inserts sutures on the beating heart, the anesthesiologist needs to meticulously monitor the ECG and, if the ECG is abnormal, guide the surgeon to correct the sutured area and avoid arrhythmias. In the present study, no cases of CAVB occurred in on beating heart surgery, although one case of CAVB was observed after the second aortic unclamping in a Group B patient. Previous studies have also suggested that repairing a VSD on a beating heart is an effective strategy for avoiding CAVB $(12,16)$. Air embolism remains a significant concern in the repair of septal defects 
on a beating heart (10); however, previous studies have reported that opening the beating left ventricle does not necessarily increase the risk of air embolism $(12,21,25)$. In light of this evidence, routine measures were applied in the present study to prevent air intake to the left ventricle, and continuous monitoring of TEE showed no significant air volume in the left ventricle. The holes in the residual VSD might have been too small, preventing air from reaching the left ventricle. Notably, our result also showed no neurological complications in Group A, which suggests that these measures were significantly effective.

Overall, our study revealed that residual shunts are likely to occur in the perimembranous portion of ventricular septal below the septal flap of the tricuspid valve, which is in a deep location and not easily exposed. In contrast, supracristal residual shunts are easily exposed and repaired due to the simple surrounding anatomical structure. We did not attempt on beating heart surgery for muscular VSD residual shunts as the location was too deep to expose.

\section{Limitations}

The present study has both strengths and limitations. A significant strength was that it collected and analyzed the data of pediatric patients that were difficult to obtain in large numbers and compared two groups of patients with similar baseline characteristics. This facilitated comparable perioperative parameters. However, as the incidence of large residual shunts during VSD repair remains low and unpredictable, prospective studies can be time consuming, and retrospective studies are comparatively easy to conduct. Additionally, due to the low incidence of residual shunts, the present study's sample size was small. Further studies that are large-scale and multi-center are required to validate these findings.

\section{Conclusions}

The findings of the present study revealed that the closure of VSD residual shunts with on beating heart surgery in pediatric patients significantly reduced the incidence of prolonged ventilation time and prolonged ICU stay, as well as in-hospital expenses. The incidence of postoperative complications has not been increased in on beating heart surgery patients, and this technique achieved similar repair results to that of conventional techniques. Therefore, the on beating heart approach is relatively safe and effective for the closure of residual shunts in pediatric patients.

\section{Acknowledgments}

We thank all our cardiac surgical team colleagues for their valuable input.

Funding: This work was supported by a grant from the Science and Technology Planning Project of Guangdong Province, China (grant number 2017A070701013).

\section{Footnote}

Reporting Checklist: The authors have completed the STROBE reporting checklist. Available at http://dx.doi. org/10.21037/atm-20-5041

Data Sharing Statement: Available at http://dx.doi. org/10.21037/atm-20-5041

Conflicts of Interest: All authors have completed the ICMJE uniform disclosure form (available at http://dx.doi. org/10.21037/atm-20-5041). The authors have no conflicts of interest to declare.

Ethical Statement: The authors are accountable for all aspects of the work in ensuring that questions related to the accuracy or integrity of any part of the work are appropriately investigated and resolved. The study was conducted in accordance with the Declaration of Helsinki (as revised in 2013), and reviewed and approved by the Institutional Review Board of Guangdong Province People's Hospital (Guangzhou, Guangdong, China) (No. GDREC2019768H) on 31 December, 2019. The requirement for informed consent was waived because of the retrospective nature of the research.

Open Access Statement: This is an Open Access article distributed in accordance with the Creative Commons Attribution-NonCommercial-NoDerivs 4.0 International License (CC BY-NC-ND 4.0), which permits the noncommercial replication and distribution of the article with the strict proviso that no changes or edits are made and the original work is properly cited (including links to both the formal publication through the relevant DOI and the license). See: https://creativecommons.org/licenses/by-nc-nd/4.0/.

\section{References}

1. Mitchell SC, Korones SB, Berendes HW. Congenital heart disease in 56,109 births. Incidence and natural history. 
Circulation 1971;43:323-32.

2. Garg N, Nayyar M, Khouzam RN, et al. Peri-procedural antibiotic prophylaxis in ventricular septal defect: a case study to re-visit guidelines. Ann Transl Med 2018;6:18.

3. Rao PS, Harris AD. Recent advances in managing septal defects: atrial septal defects. F1000Res 2017;6:2042.

4. Yin S, Zhu D, Lin K, et al. Perventricular device closure of congenital ventricular septal defects. J Card Surg 2014;29:390-400.

5. Dodge-Khatami A. Optimal exposure for closure of ventricular septal defects through the tricuspid valve. Transl Pediatr 2018;7:367-8.

6. Hanna BM, El-Hewala AA, Gruber PJ, et al. Predictive Value of Intraoperative Diagnosis of Residual Ventricular Septal Defects by Transesophageal Echocardiography. Ann Thorac Surg 2010;89:1233-7.

7. Stevenson JG, Sorensen GK, Gartman DM, et al. Transesophageal echocardiography during repair of congenital cardiac defects: identification of residual problems necessitating reoperation. J Am Soc Echocardiogr 1993;6:356-65.

8. Dodge-Khatami A, Knirsch W, Tomaske M, et al. Spontaneous closure of small residual ventricular septal defects after surgical repair. Ann Thorac Surg 2007;83:902-5.

9. Berglund E, Johansson B, Dellborg M, et al. High incidence of infective endocarditis in adults with congenital ventricular septal defect. Heart 2016;102:1835-9.

10. Thapmongkol S, Sayasathid J, Methrujpanont J, et al. Beating heart as an alternative for closure of secundum atrial septal defect. Asian Cardiovasc Thorac Ann 2012;20:141-5.

11. Menting ME, Cuypers JA, Opic P, et al. The unnatural history of the ventricular septal defect: outcome up to 40 years after surgical closure. J Am Coll Cardiol 2015;65:1941-51.

12. Ozal E, Yilmaz AT, Arslan M, et al. Closing perimembranous ventricular septal defects in adult patients in the beating heart. J Card Surg 2002;17:143-7.

13. Schipper M, Slieker MG, Schoof PH, et al. Surgical Repair of Ventricular Septal Defect; Contemporary Results and Risk Factors for a Complicated Course. Pediatr Cardiol 2017;38:264-70.

14. Becerra V, Althabe M, Salgado G, et al. Ventricular septal defect repair in children during first year of life in a public hospital. Arch Argent Pediatr 2014;112:548-52.

15. Ma J, Li XH, Yan ZX, et al. Effect of myocardial protection during beating heart surgery with right sub- axiliary approach. Chin Med J (Engl) 2009;122:150-2.

16. Ma R, Yang Z, Yingbin X. Repair of massive ventricular septal defect with tricuspid straddling under mild hypothermia cardiopulmonary bypass with beating heart. Int J Cardiol 2010;143:e19-20.

17. Day JR, Taylor KM. The systemic inflammatory response syndrome and cardiopulmonary bypass. Int J Surg 2005;3:129-40.

18. Yilmaz AT. Does direct visualization of peripatch areas in beating heart eliminate the risk of residual ventricular septal defect in adult patients? J Cardiovasc Surg (Torino) 2003;44:51-4.

19. Reber D, Tosson R, Khargi K, et al. Repair of a postinfarct ventricular septal defect on the beating heart. Surgical considerations. J Cardiovasc Surg (Torino) 2002;43:465-6.

20. Siondalski P, Jarmoszewicz K, Rogowski J, et al. Emergency surgical closure of postinfarction ventricular septal defect on the beating heart. Interact Cardiovasc Thorac Surg 2007;6:160-2.

21. Tang Y, Wu Y, Zhu J, et al. Total endoscopic repair of atrial septal defect under on-pump beating heart. J Thorac Dis 2018;10:6557-62.

22. Roos-Hesselink JW, Meijboom FJ, Spitaels SE, et al. Outcome of patients after surgical closure of ventricular septal defect at young age: longitudinal follow-up of 22-34 years. Eur Heart J 2004;25:1057-62.

23. Lin A, Mahle WT, Frias PA, et al. Early and delayed atrioventricular conduction block after routine surgery for congenital heart disease. J Thorac Cardiovasc Surg 2010;140:158-60.

24. Sahu MK, Das A, Siddharth B, et al. Arrhythmias in Children in Early Postoperative Period After Cardiac Surgery. World J Pediatr Congenit Heart Surg 2018;9:38-46.

25. Graffigna A, Minzioni G, Ressia L, et al. Surgical ablation of ventricular tachycardia secondary to congenital ventricular septal aneurysm. Ann Thorac Surg 1994;57:921-4.

(English Language Editors: B. Madden and J. Reynolds)

Cite this article as: Cao Z, Chai Y, Liu J, Liu S, Wei J, Liang J, Zhuang J, Wang S, Xu G. Revising ventricular septal defect residual shunts without aortic re-cross-clamping: a safe and effective surgical procedure. Ann Transl Med 2020;8(18):1134. doi: 10.21037/atm-20-5041 